\title{
VARIATION OF THE FIR/SUBMM OPTICAL PROPERTIES OF SILICATE DUST ANALOGUES AT LOW TEMPERATURE: IMPLICATIONS FOR THE OBSERVATIONS OF INTERSTELLAR COLD DUST EMISSION
}

\author{
K. Demyk ${ }^{1}$, C. Meny ${ }^{1}$, A. Coupeaud ${ }^{2}$, C. Nayral ${ }^{3}$, H. Leroux ${ }^{1}$, \\ C. Depecker ${ }^{4}$, G. Creff $^{1}$, J.-B. Brubach ${ }^{1}$ and P. Roy ${ }^{5}$
}

\begin{abstract}
We present here the latest results from experimental studies aiming at measuring the Mass Absorption Coefficient (MAC) of interstellar silicate dust analogues at low temperatures in the far infrared and submillimetre domains which are necessary for the analysis of astronomical data. These results show that above $100 \mu \mathrm{m}$, the MAC of amorphous silicate nano-grains has a complex dependence with the grain temperature and with the wavelength. This has to be considered when interpreting astronomical observations to avoid errors on the derived parameters such as the dust mass and its physical and chemical properties.
\end{abstract}

\section{Introduction}

The Herschel and Planck space missions are now providing abundant data in the far-infrared (FIR) to millimetre ( $\mathrm{mm}$ ) wavelength range, a spectral domain that traces cold dust and allows us to detect pre-stellar clouds prior to their collapse, and thus to study the very first steps of star formation, and to estimate a more precise dust mass in cold interstellar clouds. A good knowledge of the dust physical, chemical and optical properties, and of its thermal emission is therefore needed in order to correctly interpret observational data. The dust emission in the FIR is usually modelled by a modified blackbody spectral distribution and the dust mass absorption coefficient (MAC or $\kappa$ ) is commonly taken to vary as $\lambda^{-\beta}$ (Hildebrand 1983), with the emissivity spectral index $\beta$ ranging from 1 to 2 , a value of two,

\footnotetext{
1 Université de Toulouse, UPS-OMP, IRAP, Toulouse, France

2 CNRS, IRAP, 9 avenue colonel Roche, BP. 44346, 31028 Toulouse Cedex 4, France

3 Université de Toulouse, INSA, CNRS, LPCNO, 31077 Toulouse, France

4 UMET, UMR 8207, Université Lille 1, CNRS, 59655 Villeneuve d'Ascq, France

5 Ligne AILES - Synchrotron SOLEIL, L'Orme des Merisiers, 91192 Gif-sur-Yvette, France
} 
characteristic of amorphous materials, being commonly adopted in astrophysical studies. However more and more observational results question this model: the FIR excess (Reach et al. 1995), the anti-correlation of the dust temperature with the spectral index and estimated values of $\beta$ larger than 2 (Dupac et al. 2003; Désert et al. 2008; Paradis et al. 2010 and reference therein; Planck Collaboration et al. 2011). These results could partly be due to temperature variations along the line of sight (Juvela \& Ysard 2012) or high noise level in the observations (Shetty et al. 2009) and detailed modelling is needed to quantify the possible contribution of each effect. In addition, it is important to realise that the dust optical properties at these long wavelengths and at low temperatures are not well known and they are thus only extrapolated, in astronomical models, from those measured at shorter wavelengths and at room temperature. However, experimental studies have shown that the optical properties of dust depend on the frequency and on the temperature (Agladze et al. 1996; Mennella et al. 1998; Boudet et al. 2005) and thus such an extrapolation is incorrect (Coupeaud et al. 2011). We present here some of the latest experimental results on the FIR/submm optical properties of silicate dust analogues and discuss their implications for the interpretation of astronomical data.

\section{Experimental setup, sample synthesis and characterisation}

Nanograins analogues of interstellar silicate dust are synthesized by a sol-gel method following various recipes allowing us to control the size, composition and structure of the grains. The MAC of the synthesized analogues are measured in transmission in the $2-1500 \mu \mathrm{m}$ range at various temperature with the ESPOIRS experimental setup (Études Spectroscopiques des Propriétés Optiques InfraRouge et Submillimétrique of interstellar dust analogues) and with the setup on the AILES (Advanced Infrared Line Exploited for Spectroscopy) beam line at the synchrotron SOLEIL (Brubach et al. 2010). Both setups are composed of a Fourier Transform InfraRed (FTIR) spectrometer coupled with a cryostat which allows us to cool down the samples from $300 \mathrm{~K}$ to $10 \mathrm{~K}$ and He cooled bolometers (at $4 \mathrm{~K}$ and $1.6 \mathrm{~K}$ ) for measurements above $100 \mu \mathrm{m}$. More details on the experimental setup, sample synthesis and characterisation and results on additional dust analogues may be found in Coupeaud et al. (2011).

\section{Experimental results and astrophysical implications}

The MAC for two analogues representative of amorphous cosmic dust: one having an olivine stoichiometry $\left(\mathrm{Mg}_{2.3} \mathrm{SiO}_{4}\right)$ and the other a pyroxene one $\left(\mathrm{Mg}_{0.9} \mathrm{SiO}_{3}\right)$ are shown in Figure 1. The first observations are that the MAC of both samples decreases when the grain temperature decreases indicating that some energetic processes are activated when the temperature increases. Secondly, the MAC of both samples has a complex dependence on the wavelength (which is different for the two samples) and on the temperature: for both samples, at a given wavelength, 

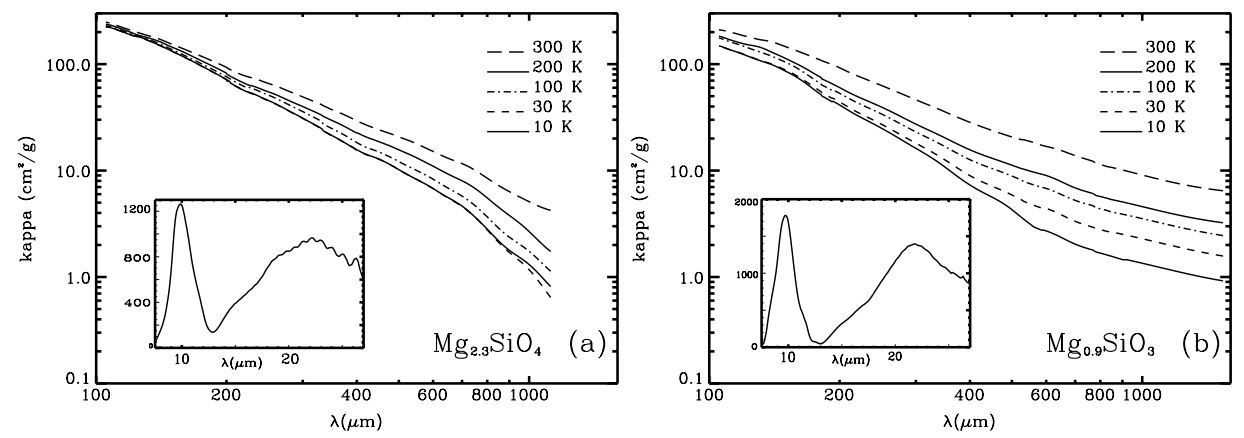

Fig. 1. Mass absorption coefficient of amorphous samples with an olivine (a) and a pyroxene stoichiometry (b), at different temperatures, in the 100-1000(1200) $\mu \mathrm{m}$ range. The inset plots show the MIR spectrum of the analogues at room temperature.

the slope of the MAC, i.e. what we can defined as the local spectral index, $\beta_{l o c}$, is anti-correlated with the temperature. We find that for $100 \mu \mathrm{m} \leq \lambda \leq 500 / 700 \mu \mathrm{m}$, $\beta_{l o c}$ is in the range $1.6-2.1$ for all grain temperatures and grain compositions whereas, for $\lambda \geq 500 / 700 \mu \mathrm{m}$, $\beta_{\text {loc }}$ may either decrease down to 1 for samples with a pyroxene stoichiometry or increase up to 3 for samples with an olivine stoichiometry. These results are in agreement with previous studies on amorphous silica $\left(\mathrm{SiO}_{2}\right)$ and $\mathrm{Mg}$ - and Fe-rich silicate analogues (Agladze et al. 1996; Mennella et al. 1998; Boudet et al. 2005; Coupeaud et al. 2010). These variations of the MAC are due to the amorphous structure of the material as shown by the fact that the MAC of the same samples, crystallised by annealing at $1100^{\circ} \mathrm{C}$, do not present any changes with the grain temperature and wavelength (Coupeaud et al. 2011). These results may be understood within the framework of models from solid-state physics that applies to amorphous material and that describe local defects and disorder on atomic scales by a single charge correlation length and an ordered distribution of two-levels systems (Meny et al. 2007) and that are able to qualitatively reproduce, the experimental results and the astronomical observations as well (Paradis et al. 2011).

These results have several implications for the interpretation of astronomical observations. The first one is that it is incorrect to describe the MAC of interstellar amorphous dust analogues with the simplified asymptotic expression $\lambda^{-\beta}$ based on a single temperature and wavelength independent spectral index, as it is often done to interpret FIR/submm observations. Doing so may introduce errors on the derived parameters, such as the dust mass but also in the physical and chemical dust properties. For example the value of the MAC measured for the $\mathrm{Mg}_{0.95} \mathrm{SiO}_{3}$ sample at $1200 \mu \mathrm{m}$ is $0.45 \mathrm{~cm}^{2} \mathrm{~g}^{-1}, 3$ times higher than the one extrapolated from $100 \mu \mathrm{m}$ with $\beta=2,0.17 \mathrm{~cm}^{2} \mathrm{~g}^{-1}$, leading to the underestimation by a factor 2.6 of the dust mass when the extrapolated MAC is used. Another important aspect is that the emissivity spectral index $\beta_{e m}$ of the same region derived from observations performed in two distinct spectral domains may be different although 
it characterises the same dust population. As a consequence, comparing spectral indexes derived from different wavelength domains is in principle not possible and this must be kept in mind when comparing $\beta$ - $\mathrm{T}$ diagrams or when flux ratios are used to derived the dust and cloud masses.

These experimental results emphasise that the interpretation of FIR/submm observations and the modelling of the interstellar dust emission should thus be based on the dust MAC of the various dust component as a function of the wavelength and temperature. The dependency on the wavelength was for example introduced to the MAC of the astrosilicates by Li \& Draine (2001) to account for the high galactic latitude dust emission measured by FIRAS. The next step is now to introduce the dependence on the temperature and this may be done with alternative physical models such as the TLS model (Meny et al. 2007; Paradis et al. 2011). In addition because interstellar dust consists in several dust components with different composition that varies from one environment to another, further laboratory experiments are needed on additional dust analogues such as Fe-rich dust analogues, oxides, carbonaceous dust, ice mantles.

We acknowledge SOLEIL for provision of synchrotron radiation facilities. This work has been supported by the French National Agency (ANR-07-BLAN-0364-01, "Cold Dust") and by the European programme FEDER and the programme "Molécules et grains : du laboratoire à l'Univers" of the Midi-Pyrénées Observatory.

\section{References}

Agladze, N.I., Sievers, A.J., Jones, et al., 1996, ApJ, 462, 1026

Boudet, N., Mutschke, H., Nayral, C., et al., 2005, ApJ, 633, 272

Brubach, J., Manceron, L., Rouziéres, M., et al., 2010, in American Institute of Physics Conf. Series, 1214, edited by A. Predoi-Cross \& B.E. Billinghurst, 8184

Coupeaud, A., Demyk, K., Meny, C., \& Nayral C., 2010, Proceedings of the Annual meeting of the French Society of Astronomy and Astrophysics, edited by S. Boissier, M. Heydari-Malayeri, R. Samadi and D. Valls-Gabaud, 205

Coupeaud, A., Demyk, K., Meny, C., et al., 2011, A\&A, 535, A124

Désert, F., Macías-Pérez, J.F., Mayet, F., et al., 2008, A\&A, 481, 411

Dupac, X., Bernard, J., Boudet, N., et al., 2003, A\&A, 404, L11

Hildebrand, R., 1983, Q. Jl R. Astr. Soc., 24, 267

Juvela, M., \& Ysard, N., 2012, A\&A, 539, A71

Li, A., \& Draine, B.T., 2001, ApJ, 554, 778

Mennella, V., Brucato, J.R., Colangeli, L., et al., 1998, ApJ, 496, 1058

Meny, C., Gromov, V., Boudet, N., et al., 2007, A\&A, 468, 171

Paradis, D., Veneziani, M., Noriega-Crespo, A., et al., 2010, A\&A, 520, L8

Paradis, D., Bernard J.-P., Meny C., \& Gromov V., 2011, A\&A, 534, A118

Planck Collaboration, 2011, A\&A, 536, A22

Reach, W.T., Dwek, E., Fixsen, D.J., et al., 1995, ApJ, 451, 188

Shetty, R., Kauffmann, J., Schnee, S., et al., 2009, ApJ, 696, 676 